\title{
Promoting Standards in Quantitative Atom Probe Tomography Analysis
}

\author{
R. M. Ulfig*, T. F. Kelly,* and B. Gault** \\ * Imago Scientific Instruments Corporation, 5500 Nobel Drive, Madison, WI 53711, USA \\ ** Australian Key Centre for Microscopy and Microanalysis, University of Sydney, Sydney, \\ Australia
}
Atom probe tomography (APT) is a maturing three-dimensional compositional technique with nanoscale spatial resolution. The instrument is a combination of a field ion microscope and a mass spectrometer and has been described in great detail previously [1]. Recent advancements in sample preparation techniques, the introduction of a commercially available laser-pulsed system, ease and speed of data collection, and advances in data analysis software have expanded the role of the technique from primarily metal systems to a wide variety of semiconductor and data storage applications [1-3].

The maturity of the technique has moved the use of the instrument out of the purely academic research laboratories and into industrial use. As a technique matures and moves towards mainstream use, it is critical to the credibility of the technique that work can be repeated and verified to be both accurate and precise over a wide variety of use cases and users. A natural progression of quantitative data analysis techniques have been developed and published in various reviewed journals over the past forty-plus years and many have been incorporated into commercially available software, but no wide-reaching group has yet formed to establish standard data reconstruction and analysis protocols. Standards are needed to cover preferred nomenclature, definitions, analysis protocols, data formats, and more.

Evidence of the need for standards can be found in the most basic quantitative use of the technique defining the overall composition in a mass histogram. Simple quantitative analysis requires the user to define ranges in a mass-to-charge histogram, subtract background, then sum the counts and compute composition. A mass-to-charge histogram was created artificially using typical peak shapes and background noise with a pre-determined number of counts for aluminum, magnesium, silicon, and copper peaks, as shown in Fig. 1. Experts in atom probe tomography were asked to provide ranging information which was then used to calculate their interpretation of the overall composition. Comparisons to the known values were shown to have differences of 5 percent or more [4]. The inability to provide precise or accurate quantitative data, even from a simple analysis underscores the need for standards in APT.

We propose that the International Field Emission Society, the leading professional society in atom probe tomography, establish an international committee working with international standards organizations to develop consensus standards for atom probe tomography.

[1] T.F. Kelly, et al., Review of Scientific Instruments 78 (2007) 20.

[2] H. Fukuzawa, et al., Journal of Physics D: Appl. Phys. 40 (2007) 1213.

[3] J.S. Moore, et al., Ultramicroscopy 108 (2008) 536.

[4] B. Gault, et al., in Atom Probe Reconstruction \& Data Analysis Workshop. (2008).

[5] Helpful discussions with D. Baer, Pacific Northwest National Laboratory are gratefully acknowledged 


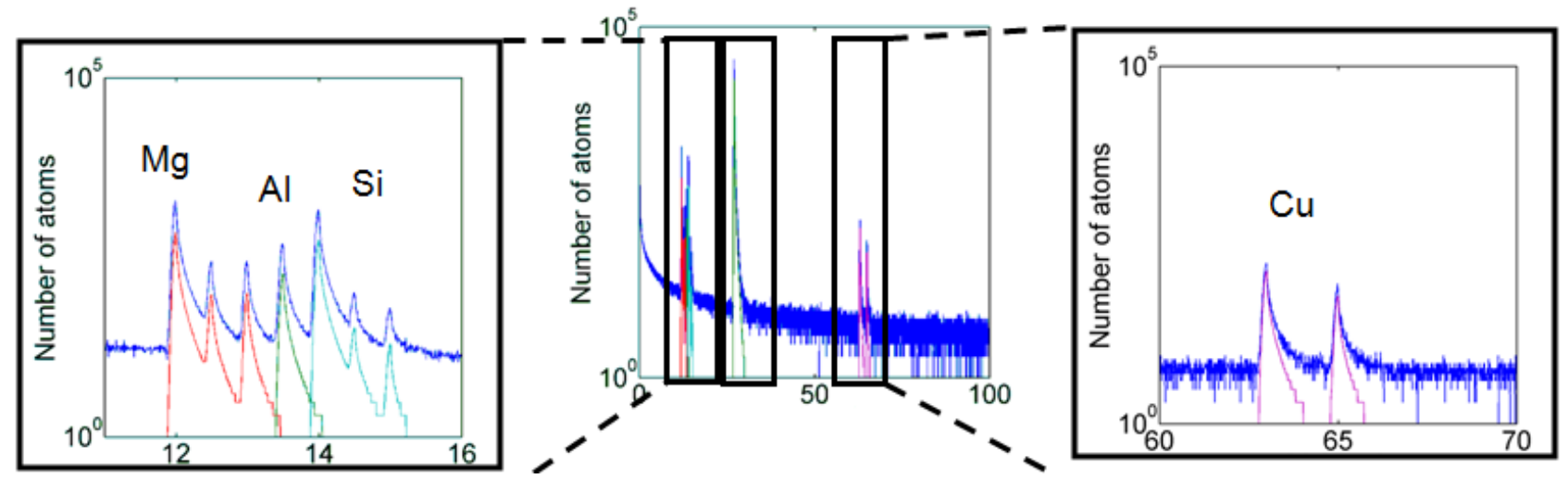

FIG. 1. Artificial mass-to-charge state histogram of an aluminum, magnesium, silicon, copper alloy with a known number of atoms in each of the peaks.
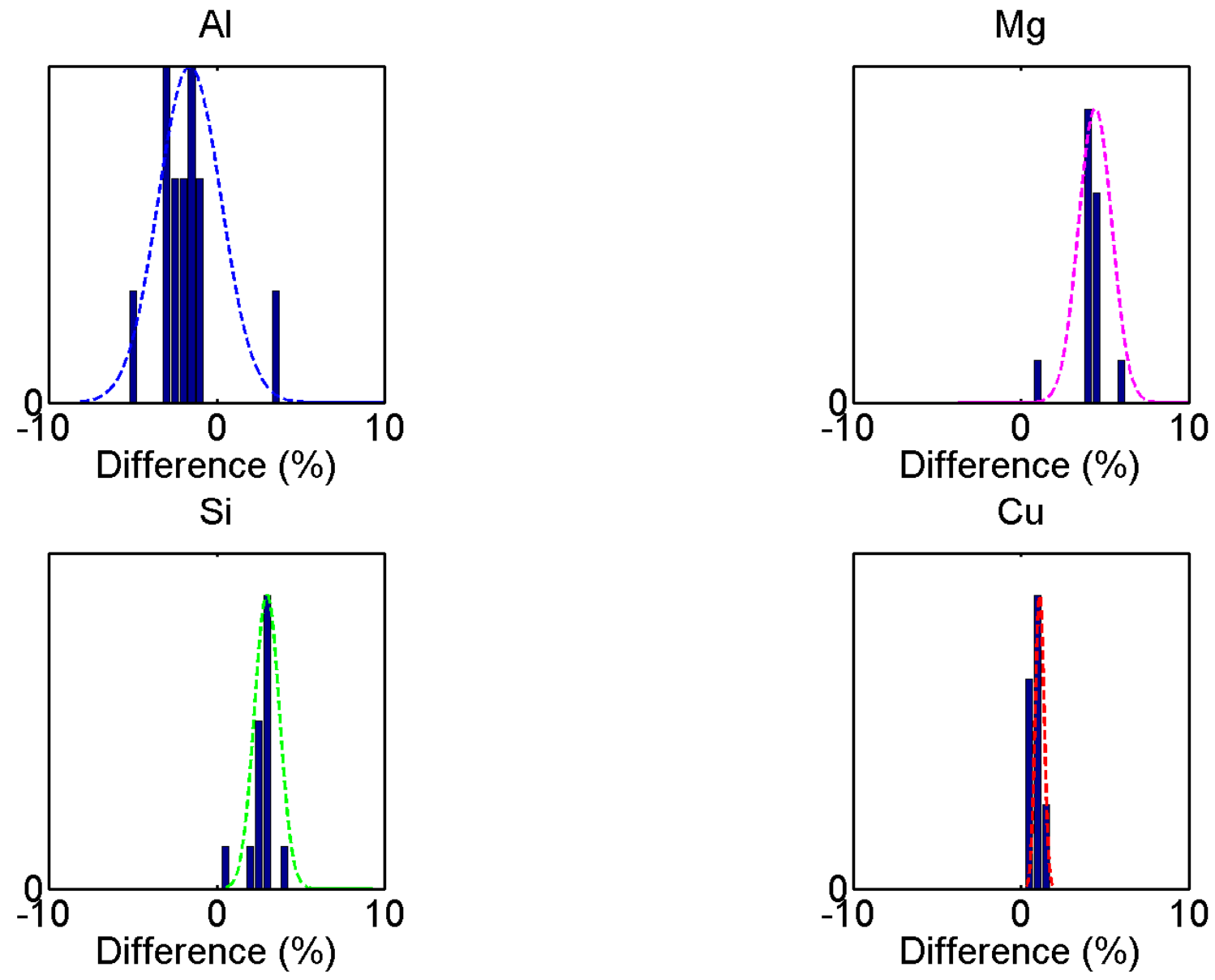

FIG. 2. The difference of defined ranges and compositional calculation for each of the species in the artificial alloy spectrum from the actual known counts. 